\title{
USER INTERFACES FOR INFORMATION SYSTEMS
}

\author{
N. J. Belkin", P. G. Marchetti**, M. A. Albrecht*", L. Fusco**, S. Skogvold**, H. \\ Stokke*", G. Troina**
}

El siguiente artículo nos ha sido remitido por el director de ESA-IRS, Mr. Marino Saksida. Es el resultado del seminario interno ESRIN sobre "User interfaces for Information Systems" celebrado en Frascati, Italia, el 23 de febrero de 1990. Fue publicado en ESA, Journal, vol. 14, 1990.

Lo consideramos de suficiente interés como para reproducirlo en esta revista, aunque dada su especialización temática, hemos optado por suprimir el apartado 3.2 del trabajo original referido a «Earth observation user services requirements». Con esto creemos interpretar los intereses de la mayoría de nuestros lectores.

\begin{abstract}
This paper presents descriptions of four information-system interface projects in progress at ESRIN, each demonstrating a somewhat different approach to interface design, but all sharing the commonality of responding to user goals, tasks and characteristics. It is suggested that next-generation scientific information systems will have to be designed for direct access by end users to a large variety of information sources, through a commom interface. Design of such systems, including their interfaces, should be based on a multi-level analysis of user goals, tasks and domain views.

Keywords: Information-system interfaces, interface design, direct access interfaces.

Resumen: Se describen cuatro proyectos de interfaces de sistemas de información que se están desarrollando en ESRIN (establecimiento de la Agencia Espacial Europea, en Frascati). Cada uno de ellos muestra un enfoque diferente del diseño de interfaces, pero todos tienen en común el responder a los objetivos, tareas y características de los usuarios. Se sugiere que la próxima generación de sistemas de información científica se tendrá que diseñar para permitir el acceso directo de los usuarios finales a una gran variedad de fuentes de información a través de una interfaz común. El diseño de tales sistemas y de sus interfaces debería basarse en un análisis multinivel de objetivos, tareas y puntos de vista propios de la materia de trabajo de cada usuario.
\end{abstract}

Palabras clave: Interfaces en los sistemas de información, diseño de interfaces, interfaces de acceso directo.

\section{Introduction}

The issue of information support for the accomplishment of a variety of goals and associated tasks has led to the construction of a large number of different kinds of computerised databases and mechanism for accessing them, i.e., «information systems". Examples of such systems include scientific data banks, bibliographic information-retrieval systems, and management information systems.

Traditionally, such information systems have been constructed on the basis of two underlying assumptions: firstly that each different kind of database requires a different, and separate, information-system structure; and secondly that those who access such systems will be specialists in the use of the particular system with which they interact. These assumptions have led to highly specific information-

* School of Communication, Information \& Library Studies, Rutgers University, New Jersey, USA.

** ESA/ESRIN, Frascati, Italy.

Recibido 30-1-91. 
system designs in terms of database structures and organising principles, retrieval mechanisms, and interfaces. These designs are tailored to particular kinds of data, and to efficient retrieval on the basis of complex query construction using elaborate command languages.

Recently, both of the assumptions on which such systems are based have been called into question. The former, because it has become clear that achievement of any one goal and its associated tasks, which might require information support, often requires different kinds of data or information; the latter, because the people who are performing the end tasks wish to access the information systems directly, rather than through specialist intermediaries. These challenges imply very different kinds of information-system design than have been experienced to date. Specifically, information systems that respond to these challenges would allow "seamless" access to the range of information sources (databases) relevant to particular goals and tasks, via interfaces that not only allow, but actively support, direct end-user interaction.

If these challenges are accepted, then new information-system design principles and methods must be developed. In particular, answers to the questions of:

- how to determine the relevant information sources for a given context;

- how to integrate (or allow uniform access to) the required variety of information sources, and

- how to design interfaces for end-user support

are of paramount importance.

One way to address these questions is to consider seriously the overall function of the information system that is to support the users of the system in performing the tasks that will help them to realise their goals. This suggests that we approach information-system design from the point of view of the users; specifically, to determine how the users' views of their domain, goals and tasks might specify the range and relationships of relevant information sources, and the most appropriate means of access to them. This is, in effect, a prescription for user goals and task analysis as the basis for all aspects of information-system design.

Seen from this point of view, it becomes clear that interface design should be embedded in whole-system design, rather than tacked onto some unrelated, predefined structure. Indeed, it appears that considering the issue of the interface as an active support tool, allows the questions raised above to be addressed within an integrated framework. This approach to the information-system design problem turned out to be a central theme of the recent ESRIN Workshop which, although focussed on specific user interfaces, had broader implications.

The following sections of this paper report on some specific interface projects being carried out at ESRIN, in a variety of contexts. Each demonstrates a somewhat different approach to interface design, due to their individual contexts, but all share the commonality of responding to user goals, tasks and characteristics at various levels. The methods of analysis and representation of goal, task or domain are also sensitive to the specific context in which they are applied.

The first two projects reported on, the European Space Information System (ESIS) and the Expert System for Accessing Remotely Sensed Data (ESARS), 
focussed initially on high-level user goal analysis and domain representation as the basis for their system design. This was required because the issue of heterogeneous data types is highly significant in the areas of concern, and was possible because both projects were able to identify classes of relatively homogeneous user communities.

The Intelligent Interfaces for Bibliographic Information Retrieval (IR) project uses a lower-level Cognitive Task Analysis (CTA) specific to the informationretrieval problem or situation. This is because users in this context cannot be classified by subject domain, but they share the common goal of finding relevant information through interaction with an information system.

The final two systems reported on, HANDYNET and QWIN, address implementation details of interfaces to specific databases or with specific database views. The implementations are derived, in these cases, from considerations of user tasks and characteristics in the specific circumstances of given databases and access mechanisms.

In this introduction, we have focussed deliberately on the questions of goals, tasks and domain analysis as being unifying themes to all of the projects described. However, the projects also differ from one another in substantial ways. We see this as evidence of the richness of this general approach, which leads to a variety of context-dependent solutions to the problem of interface design for information systems.

\section{User interfaces for science information systems}

This section describes the work currently being undertaken to define and develop a "User Shell» for the European Space Information System (ESIS), concentrating on the user-interface aspects of the project (for further information, see Refs. 1 and 2).

ESIS is aimed at providing the space-science community with a set of tools to access, exchange and handle information from a great variety of sources, including space-mission archives, "white-» and "yellow-page» directories, and other support databases and services. In particular, access to all databases contained in ESIS must be possible through a homogeneous interrogation and data-managing language. This language will support queries formulated in "discipline-oriented» rather than "computer-oriented" terms. A pilot project running in the 1988-1992 time frame will demonstrate the validity of the concepts used and will establish a basic infrastructure for further developments.

In order to model the way in which scientists from different domains see and access their information, semantic networks have been designed that constitute the basis for the eventual retrieval system. A semantic network is a set of nodes and arcs playing, respectively, the role of concepts and the relations between them. Since each discipline has its own semantic denominations, one such network has been worked out for each of the disciplines that ESIS will serve: astronomy \& astrophysics, and space physics. Figure 1 shows the current status of the astronomical layout. The actual design of these schema has been conducted by centres of expertise in the domains of concern, under contract to ESA, and has involved the participation of users through both workshops and working groups. 


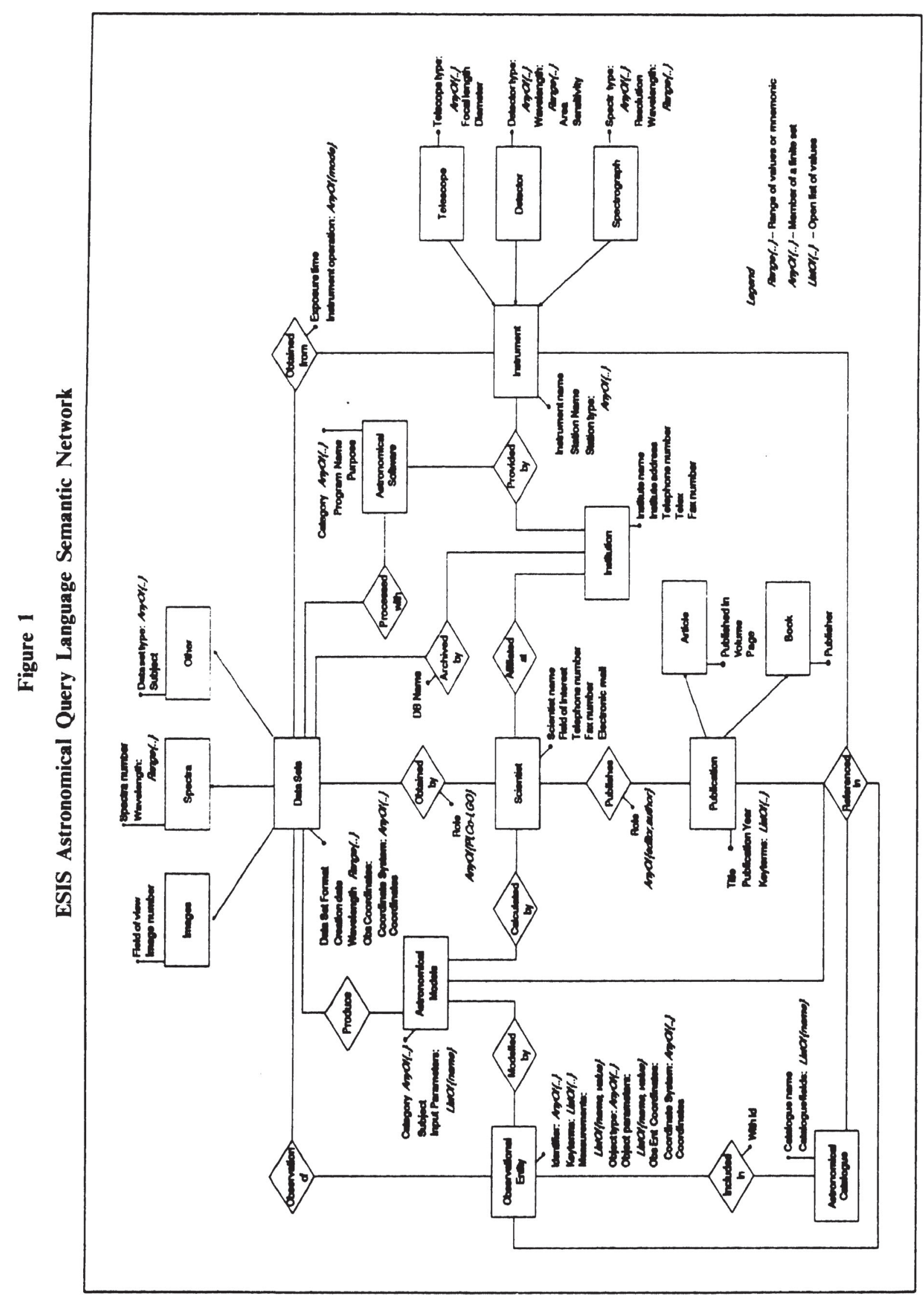




\subsection{User needs}

The general requirements of space in terms of information systems can be summarised under the following headings:

\section{a) Open existing space-data holdings to non-experts}

Due to the high level of specialisation required to access current archiving facilities, data is being utilised only by small "mission-specific" user communities. A global reference database could provide non-experts with an overview of available data. This requirement calls for an integrated data view model and access capabilities.

\section{b) Integrate past and future mission archives}

Existing and future archiving facilities should be integrated into the system without losing their identity and/or independence. For past missions, this means that current investments are preserved and that local user communities are not concerned by the integration. For future missions, independence from the system means the flexibility to set up facilities taking advantage of state-of-the-art technology without making compromises. This requirement calls for an open architecture.

\section{c) Integrate data retrieval with information exchange}

Finding out what data sets are of relevance for a particular research project, retrieving these data sets, processing them and possibly exchanging them with other collaborators, should all be activities integrated into a single environment. Provision should also be made to interface in a transparent manner with other information systems and telecommunications networks. This requirement calls for a uniform access method to all services available through ESIS.

\subsection{The ESIS User Shell}

Access to the system will be through a "User Shell», which will be distributed to end users for installation on their local computing infrastructure. The user interface is designed to support a large number of input devices, including terminals, work stations and personal computers, and offers a selection of dialogue modes such as menus, commands and forms. A user activating the User Shell will be able to reach all ESIS functions via a uniform view of the system, independent of the local computing environment and of the networking method of accessing ESIS. Online help and tutorial services will be provided locally, in addition to any similar services offered by the data facility, but not slowed by network delays. An ESIS Pilot Distributed System will provide information services such as electronic mail, bulletin boards, file transfer, etc., as well as a transparent message-passing service for ESIS applications. The ESIS Query and Correlation Environments will provide information-retrieval, handling and storage functions, as well as the possibility to cross-correlate data from different sources. 
In general, we envisage that, through the Query and Correlation Environment, users will have access to the global information contents of ESIS as well as access to personal databases. The personal databases could contain data imported from external sources, or data retrieved from the global database. The exchange of databases with other users will also be supported.

The user will interact with the environment through a suite of so-called «interaction paradigms». These paradigms will support command or query input in a variety of ways and will display results in a form suitable to the data. Personal "views" of databases will allow a customised access to the information stored. Globally pre-defined views will allow groups of individuals to share the same means of accesing data. The formulation of queries will support the combination of any of the interaction paradigms with any user-defined view. The overall environment will be enhanced by including both standard support tools and disciplinespecific ones such as coordinate-system transformations and physical-unit conversions.

Among the functions that will be available through the User Shell, the following merit highlighting:

\section{a) Query and command formulation}

Command or query formulation will be supported through a set of input paradigms that can be used in any combination at any given time.

Depending on the kind of input required, the most suitable method will be selected either by the user himself, or by the application as the default setting. Entity-Relationship (ER) diagrams will be used as the input paradigm for all functions related to the manipulation of database structure, i.e. view manipulation and management of personal databases.

\section{b) Object (data) visualisation according to structure}

The display of information items will be driven by their internal structure; in this way a vector plot will be visualised as graphs, while database records could be displayed as a form. Database structure will be shown as ER diagrams as the default. Using an object-oriented terminology, we can say that sending the message "display yourself" to any information item will produce a visualisation of the object's contents in the form best suited to that object's structure.

\section{c) Personal views and databases}

A basic requirement in a scientific information system is the ability to manipulate data according to the needs of a research project. The basis for such a capability is implemented in ESIS both through capabilities to keep and manage personal databases and capabilities to look at the database's contents in particular ways (views) that are created ciroumstantially. A graphical-concept manipulation language (3) will allow user to create and manage databases and views in a userfriendly way. 


\section{d) Information exchange}

The possibility to export data from the ESIS environment to external applications, as well as the complementary import function, will enhance its overall functionality. A set of standard external formats will be supported, such as the formats in use by the astronomical community for spectra and images (FITS) and formats for graphical metafiles. Furthermore, fully integrated support functions like mail and data-set transfer will allow the transparent exchange of data sets, personal databases, and other processed information.

\section{User interfaces for Earth observation}

The interface between the user community and the Earth Observation (EO) service providers is a key element to be considered in the preparation of future EO missions. In fact, the complexity of future payloads compared with those of current EO missions represents a major innovation in terms of products, complexity of processing, and new services. At the same time, a major international effort is presently being conducted in such areas as: product standardisation, inter-operability of regional and nationally offered services, and improved communications.

The currently offered $\mathrm{man} / \mathrm{machine}$ user-access interfaces are based on a priori knowledge of the service needed (i.e. "I want the SPOT image of the area around a given latitude and longitude and taken during a given time period»), and the information exchange is based mainly on simple communication devices like telephones or alphanumeric terminals. The availability of many instruments flying on the same EO platform will require a deep knowledge of the mission, and it is reasonable to assume that user interfaces will be based on dedicated terminals (work stations) with specialised software packages.

\subsection{User-needs evaluation by an artificial-intelligence approach}

In an interactive system a correct understanding of user requests plays a central role in supporting satellite data access. Automatic deduction of related needs, such as instrument requirements or data constraints, is also necessary. A "proper» representation for such an environment enables the user to obtain satisfactory services with a minimum of effort and enhances the quality of the information produced, in terms of efficiency and adherence to attend performance. Artificialintelligence methodologies offer new tools in modelling knowledge both on user requests and needs.

A model of the Remote Sensing (RS) domain is part of this knowledge, as well as the capacity to support high-level natural-language-based interaction. Semantic networks are a straightforward method in structuring such knowledge.

In modelling an interaction, understanding user needs implies exploring the semantic relations involved. They may often be implicit in the natural-language sentences used. The deductive process is able to acquire such implicit information and to represent a whole set of user needs. The semantic net structure, used in the 
knowledge base of ESARS (a prototype of an Expert System of Accessing Remotely Sensed Data) $(4,5)$ is described in Figure 2.

Figure 2

ESARS knowledge-base representation (simplified)

\begin{tabular}{lll} 
& \multicolumn{1}{c}{ PAYLOADS } & \\
RS & PRODUCTS & \\
APPLICATIONS & & GROUND \\
\multicolumn{1}{c}{ RS PARAMETERS } & & FACILITIES \\
RS TECHNIQUES & &
\end{tabular}

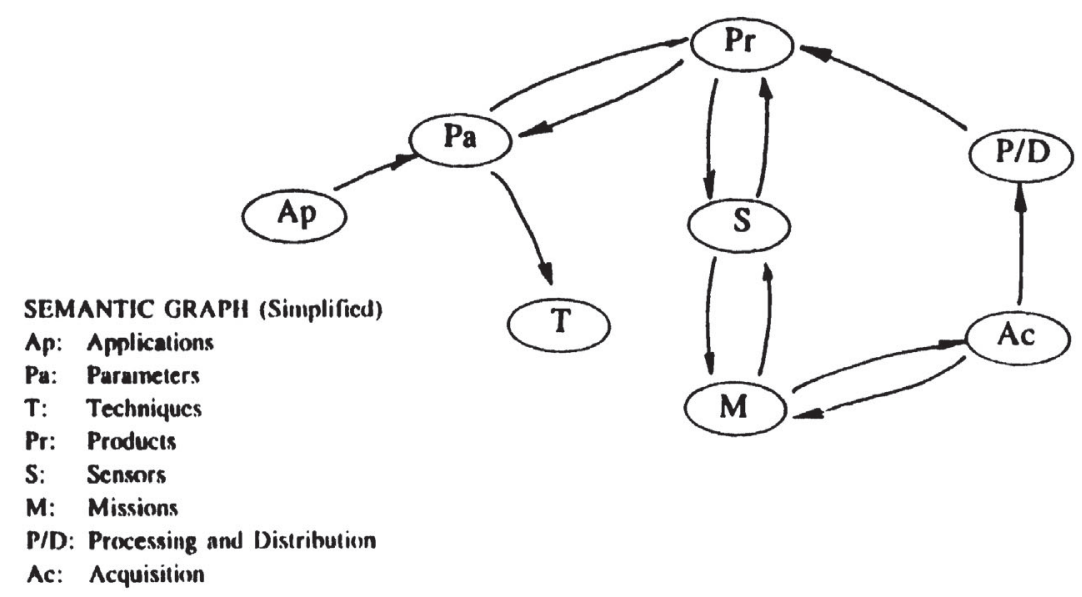

The semantic network in Figure 2 represents a generalised user view of the domain of remote sensing, its facets and their relationships to one another, which can be considered equivalent to a representation of a general Cognitive Task Analysis (CTA; see Section 4.1.2) for remote-sensing information.

The comprehension of a user query produces a proper path through the semantic graph. The inferential mechanism deduces a description of user needs as missions, instruments or data, by recursively visiting the graph's nodes. Moreover, an inverse visiting approach enables possible applications of existing data or instruments to be suggested to the user.

ESARS experience shows that the remote-sensing domain can be modelled to support very-high-level access to data, satisfactory performance and optimised data traffic.

\section{Intelligent interfaces for bibliographic information-retrieval systems}

\subsection{A design sequence for intelligent interfaces}

\subsubsection{Definition of three-stage approach to intelligent IR support}

The attempt to provide truly intelligent support for the end-users of Information Retrieval (IR) systems will require several generations of interface implementa- 
tion. The principles of formative design, as suggested by Egan (6), are appropriate in our situation; i.e. there are some ideas about the bases on which such a system should be built, but many details of the implementation are unclear, as are the effects on performance of specific aspects of our general system design.

This strategy for implementation poses some problems. The most important of these is that of maintaining consistency from generation to generation; i.e. making sure that any one implementation actually fits into the overal «ideal» interface. To this end, we suggest the following three-stage model toward intelligent information support.

Stage one: efficient end-user support. At this stage, we anticipate only that the interface masks the underlying query language, and that the knowledge contained in the interface, which is used to direct the interaction with the user, is primarily knowledge of the structure of the system. At this stage, the interface maintains static models of, for instance, the user and the system's functions.

Stage two: knowledge-based end-user support, builds upon the first by incorporating a model of what constitutes a search strategy, and incorporating knowledge of system functionality within the more flexible search-strategy functionality. At this level, more active support is offered to the user in the search-formulation process, based on knowledge of the topic of the search, as well as of the system functionality. User models will probably be adaptive in certain respects.

Stage three: intelligent end-user support will move to adaptive and interactive support of the user at the search-session level. By this we mean that the interface will be able to adapt its functions and operations to what it has learned of the user during the course of the current search session. At this stage, the interface's knowledge will be of system, topic, and user, and it will construct and maintain models of all of these, which will change during the course of the session, as required, and will be used to guide the interaction and to provide support to the user in search formulation and evaluation.

\subsubsection{Performing a CTA for information retrieval}

In order to identify the required functionality of our interface, we begin with a CTA (Cognitive Task Analysis) (7) for information retrieval, especially from the points of view of the user and the intermediary (whether human or computer) to the database. This defines the goals and activities that form the basis for domain representation such as the semantic networks of ESIS and ESARS. The difference in the IR case is that no specific subject domain can be defined in advance, so that the goal analysis is for IR in general.

In order to establish a CTA, it is necessary first to establish an overall goal for the system. In the IR system setting, we can do this by defining a hierarchy of goals, and then specifying the level (or levels) of goals that we wish to attain. The goal hierarchy that we propose for IR is specified in Figure 3, taken from Reference 8 . 
Figure 3

A goal hierarchy for information retrieval

$\begin{array}{ll}\text { LEVEL } & \text { GOAL } \\ 1 & \text { USER LEAVES THE SYSTEM } \\ 2 & \text { USER IS SATISFIED } \\ 3 & \text { APPROPRIATE RESPONSE TO USER } \\ 4 & \text { SYSTEM IS INAPPROPRIATE OR, } \\ & \text { APPROPRIATE INFORMATION }\end{array}$

EFFECTIVE SEARCH FORMULATION

The overal goal of the IR system (the level-one goal) is that the user leaves the system. This is because the IR system is a support mechanism for users, to which they should have recourse only when their own resources are inadequate for responding to some other problem or goal.

The user may leave the system for a variety of reasons, including frustration, boredom or satisfaction. In particular, we wish to consider that the level-two goal is that the user leaves the system because he or she is satisfied.

One way to achieve the level-two goal is to ensure that the user obtains an appropriate response from the rest of the system. This is the level-three goal.

One means of achieving the level-three goal of an appropriate response is to offer the user information appropriate to the situation. Another, for instance, is to suggest to the user that the particular system with which he or she is interacting is not appropriate to the user's situation. It is at this level, i.e. level 4, that we can begin to consider IR system functional design, and to specify overall goals to be achieved by the system.

The normal method for achieving the level-four goal is the construction of an effective search formulation. We can define our level-five goal of effective search formulation by specifying the tasks that are required in order to accomplish it. For this, we take the general Distributed Expert-Based Information System (DEBIS) model $(9,11)$. These level-five tasks (or goals), outlined in Figure 4 (see Ref. 8), are the activities on which the user and intermediary collaborate in order to achieve an effective search formulation.

The accomplishment of these tasks results in an evolving representation of a search topic and strategy in the context of an online IR system. It begins with the choice of interaction mode (DM) (having assumed relevance of the system to the user's situation (PM)), and then moves to general topic identification (PD). This result is used to define a context for specific search-topic, query, and searchstrategy formulation (RS), and also as a means for database selection (RS). Once databases have been selected, specific search-topic formulation begins (PD). This 


\section{Figure 4}

A problem structure for information retrieval

Level 5: Efrective Search Formulation

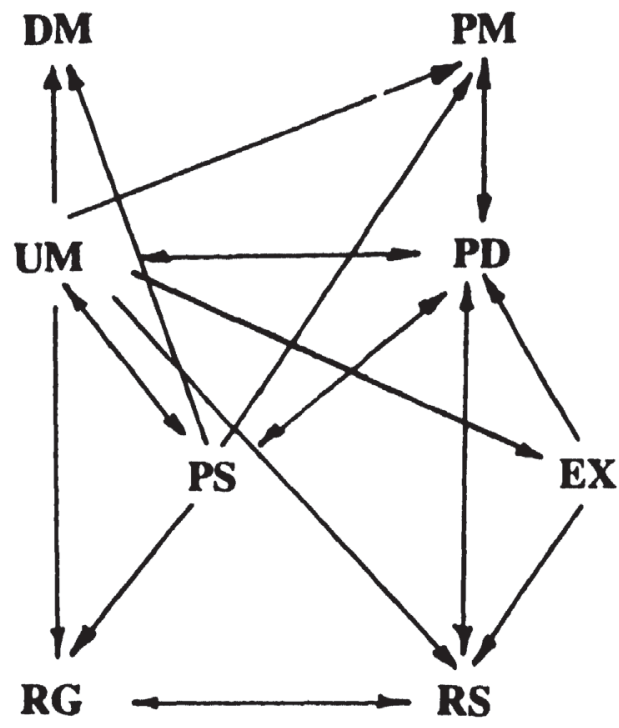

PM: Problem Mode

DM: Dialogue Mode

UM: User Model

PD: Problem Description

PS: Problem State

EX: Explanation

RG: Response Generator

RS: Retrieval Strategies

Arrows indicate logical (empirically established)

and temporal relations. General

sequence of events is from top to bottom, with iteration and recursion.

provides a basis for query specification, search-strategy formulation, and constraint identification (RS), and is also used, as required, to reformulate the general search topic (RG), or to make new decisions concerning database selection. The result of query, strategy, and constraint formulation leads to a search (RS) whose results are used to support reformulation of any of the decisions that have led to that search. The whole process is iterated to completion, as decided by the user, the reformulation decisions at any point being based on evaluation of system response in terms of user requirements.

\subsubsection{Specifying system functionality}

We specify host-system functionality in order to establish the basic operations and resources available to the host and, eventually, interface. In the following, reference is made to EsaQuest, the information retrieval system of the Agency's Information Retrieval Service (ESA-IRS).

EsaQuest has the standard host structure of inverted indices to its records, with all that this implies. In particular, this gives substantial statistical characterisation of its databases. Its resources include structured textual descriptions of its resident databases, and online thesauri for some of them. EsaQuest has a number of predefined subject clusters of databases, and a facility for user-defined clusters, both types of which can be searched in cross-file mode.

\subsubsection{Identifying constraints on the interface}

The nature of the users of ESA-IRS, and its general environment, combined with precepts for human/computer interaction in information systems, have a 
number of implications for interface design and features. The following merit special mention:

- the need to maintain a variety of access/interaction modes, to respond to the variety of users;

- the significance of intelligent explanation facilities, again in response to user variety;

- the ability to maintain long-term models of system users;

- general interface flexibility, in terms of tolerance of a variety of databases and search topics;

- support for effective interaction, rather than merely for efficient or quick searching;

- distribution of aspects of the interface between host and local-access computers;

- portability and generality of direct end-user interface software (for mounting on local machines);

- window-based interfaces, with a variety of interaction modes, including direct manipulation, menus, forms, graphics, quasi-natural language and command languages.

\subsection{Stage-one interface implementation}

Our design specification procedure, as outlined above, suggests a general structure for a level-one implementation of an intelligent interface to EsaQuest. Here, we outline the characteristics of such an interface, and then offer an example of what implementation of this interface in support of one kind of activity would look like.

The intelligent interface would be one of several different interfaces available to the system, for choice by the user or on the recommendation of the interface (at any point in the interaction). These are the native command language (EsaQuest); a form-filling menu interface based on a standard document template (see Section 6); and an interface for search-formulation support. The first of these is the current access mode, and the second is at present an internal prototype. This multiple interface structure responds to the variety of user preferences and IR experience, and also responds to some extent to the Dialogue Mode (DM) goal.

One aspect of an intelligent interface is to support terminology choice in problem description. We propose to implement this type of support by means of an interactive tool able to browse the thesaurus "before» any search action, when no set has yet been retrieved. In existing large information-retrieval systems, there is often a thesaurus available, but its accessibility and visibility are rather difficult for inexperienced users, especially when it is necessary to specify a term in the thesaurus itself in order to activate it. The browse function will have the ability to find thesaurus entries conceptually related to terms or phrases not in the thesaurus, and to navigate the thesaurus hierarchy from these entries.

Since our environment (EsaQuest) entails mainly use of large bibliographic databases, our approach has been to use statistical techniques by exploiting term 
co-occurrences and conceptual relations created by the database indexers. If the term (or noun phrase) the user is entering is not in the thesaurus, then a sample of the documents containing that entry is examined. The controlled terms indexing the documents in the sample are ranked according to their frequency in the sample of documents. The top five controlled keywords that are also thesaurus entries are shown to the user. In the first implementation of the interface, no natural-language processing is performed. From this point, user-driven navigation of the thesaurus proceeds. A full description of this interface design is presented in Reference 1.2.

\section{Multilingual menu-driven interface}

A multilingual menu-driven interface for online information retrieval has been developed by ESA-IRS. This work has been carried out in the framework of the HANDYNET project, the purpose of which is to supply information useful to handicapped people throughout the European Community. However, most of the concepts that have been used are independent of this particular application and of the information that it uses. The main characteristics of the interface, which has already been developed and is now entering an operational phase, are:

- online access to EsaQuest information and facilities;

- multilingualism;

- menu-driven access.

The first characteristic is related, of course, to the need to link users all over Europe. The interface therefore has to provide a suitable telecommunications interface. The second characteristic is related to the fact that, within the European Community, there are nine different languages and, consequently, it must be possible to access the system using any one of them. The third characteristic is the result of a choice between different methods of accessing the system in a userfriendly manner.

\subsection{User interface}

The user interface provides multilingual user-friendly access to four components:

- information retrieval;

- electronic mail;

- file transfer;

- data input.

The information-retrieval component enables menu-driven access in nine languages to two EsaQuest databases containing information mainly related to tools for handicapped people. The electronic-mail component enables friendly access to the QUEST-MAIL system, and can also be used in nine different 
languages and without any prior knowledge of the standard commands. The filetransfer component allows friendly access to the KERMIT functions «Download» and «Upload».

The data-input component enables the entry of data that will be transferred into the EsaQuest archives.

\subsection{Information-retrieval component of the user interface}

The purpose of a user interface is to make it easier for the user to access the system. The use of a system requires a certain amount of knowledge, which has to be "distributed» between the user and the user interface. Consequently, the greater the knowledge embedded in the user interface, the smaller is the knowledge that the user needs. The equation is simple:

Global knowledge $=$ User knowledge + User-interface knowledge

The global knowledge can be classified in the following way (13):

- system knowledge: about command language and available facilities (from logging-on to printing results);

- searching knowledge: about strategies and tactics involved in the searching process;

- subject knowledge: about the subject of the search;

- user knowledge: about each individual user (previous searches, preferred journals, etc.).

The menu-driven information-retrieval component of the user interface includes a certain amount of the first three kinds of knowledge (Fig. 5). With suitable selection of options on the menus, the interface generates the related EsaQuest commands. In this way, the user can access the system without knowing about EsaQuest commands, and is guided by the content of the menus towards the goal of his or her search (Fig. 6). The menu-driven approach that has been used in this interface is not the only possible approach; the query-by-form and the naturallanguage approach, for instance, are two alternatives. However, the two main advantages of the menu-driven approach are:

- only valid commands can be generated by the interface since these commands are determined in advance for each option selected;

- users can usually recognise and understand more concepts and terms than they can list; psychological studies show, in fact, that a user has a much larger passive vocabulary than an active one (14).

The major drawback of a menu-driven system is that expert users can find it slow because they have to work through a series of menus, when they would probably prefer to use a concise command string. In order to cope with different kinds of needs, this interface supplies a different dialogue mode in which the user 


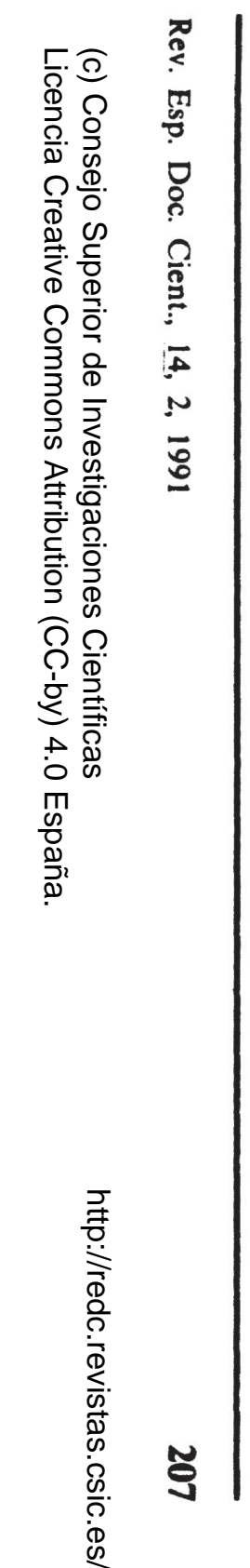

Figure 5

HANDYNET: example of menu hierarchy

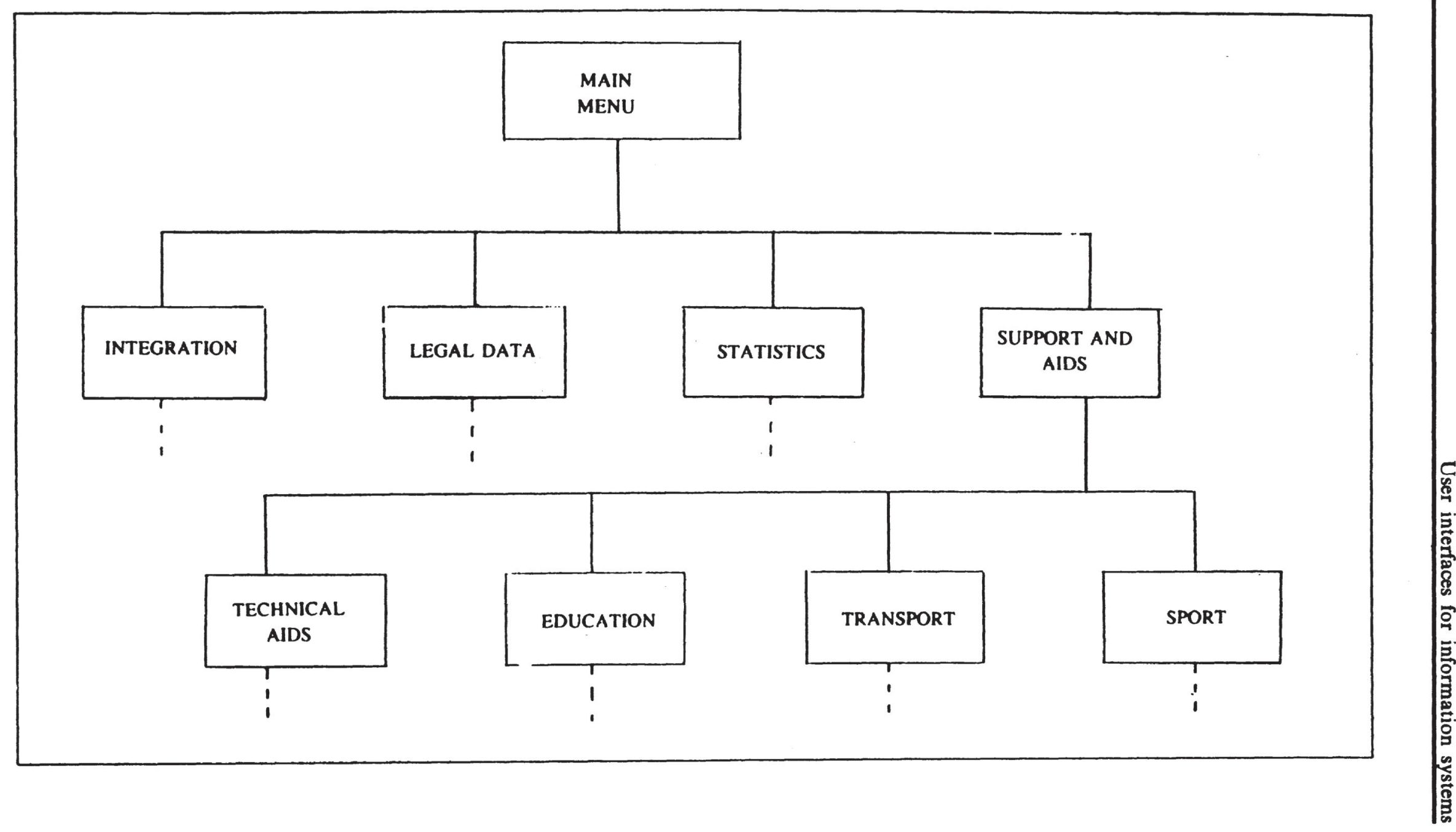


Figure 6

HANDYNET: a Greek menu

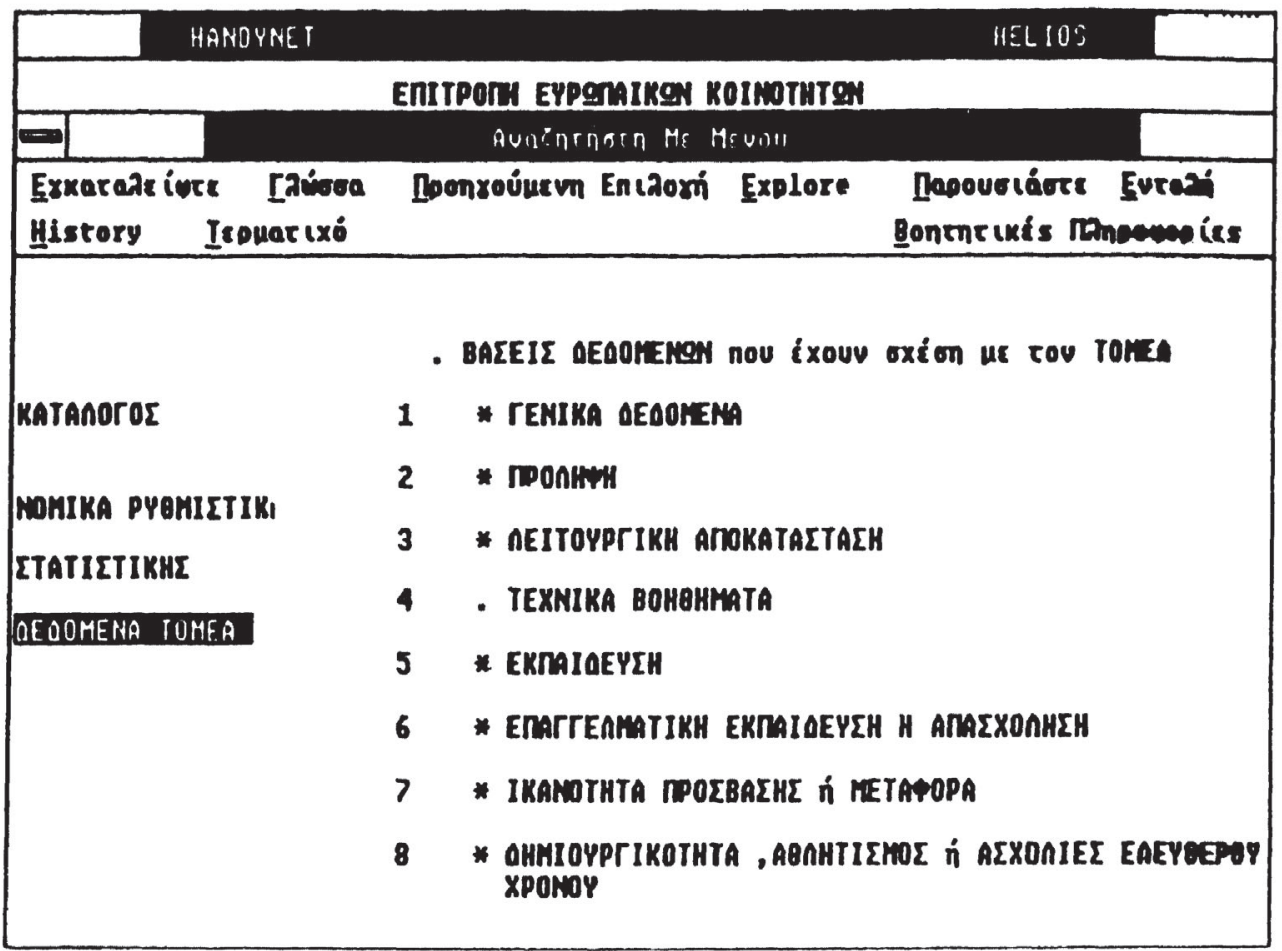

can write the content of the request. Moreover, the user has the possibility of circumventing this interface completely and accessing the telecommunications interface directly.

The interface developed by ESA-IRS for the HANDYNET project has shown the feasibility of a user-friendly interface that helps in the task of searching for information in EsaQuest databases. Some of its characteristics are of general interest (online access, multilingualism), while others are more application-related (menu-driven approach). The interface also provides some degree of adaptability in order to cope with the needs of different kinds of users.

Even though the system described has still to undergo a long period of user testing, there is every likelihood that it will form a platform in the future for the development of more complete, sophisticated EsaQuest user interfaces.

\section{A query-by-form approach to an information-retrieval interface}

This section describes QWIN, a user-friendly interface to the EsaQuest system. The target user group for QWIN includes end users who have little or no experience with online systems in general or EsaQuest in particular. The goal was to create an easy-to-use practical tool enabling the user to perform basic searches on EsaQuest without having to first learn the EsaQuest search language. It was foreseen in the requirements that the system should not require any kind of specialised help in formulating the search queries from, for example, a librarian. 
The functionality of QWIN is merely outlined here with special emphasis on the use of "Query by Forms" to present the searchable fields of the database and to enable the user to formulate search queries. The reasons for using this approach and the advantages and disadvantages of the method are discussed, and possible enhancements to the current system are presented. Finally, an alternative approach that may complement the current one in future versions of QWIN is presented.

\subsection{Why «Query by Forms»?}

The forms-based query language employed in QWIN was chosen for several reasons:

a) The user does not have to remember the prefixes or suffixes for the different fields.

b) Contrary to a menu-driven approach, the user sees the full query on the screen and is able to change any part instantly, although limited by the number of fields that are available at the time.

c) The Microsoft Windows environment is well suited for this kind of presentation; e.g. terms from retrieved documents may be pasted into any of the fields in the form.

d) In general, it is said that: «a forms-based query language allowing simple combinations of criteria composed of constant values is easier to learn and use than a command-based language (15).

e) The choice of the QBF approach was influenced by other proposed systems (16), and by commercial packages such as Personal Librarian, which uses a similar approach.

\subsection{An overview of QWIN}

QWIN begins with an automatic connection procedure, followed by a database choice window. Once a database has been selected, the user is presented with an initial search window (Fig. 7), which is the basic form of the system. This window contains various command push buttons to support the search.

In the search window, there are two basic field types, free text and fixed text, each with a pop-up menu which is displayed when a field is chosen (Fig. 8). The user can choose from these menus to operate upon the field input. When the user has completed the search-window form, the "Search" button is invoked, and the titles of items in the retrieval set are displayed in a separate window.

\subsection{Extensions to the QBF approach}

There are alternatives to the use of forms and the current QBF method should be complemented with one that overcomes basic limitations of the form approach. The use of a form or template can be discarded and the user presented with an 
Figure 7

QWIN: Query-by-Form search menu

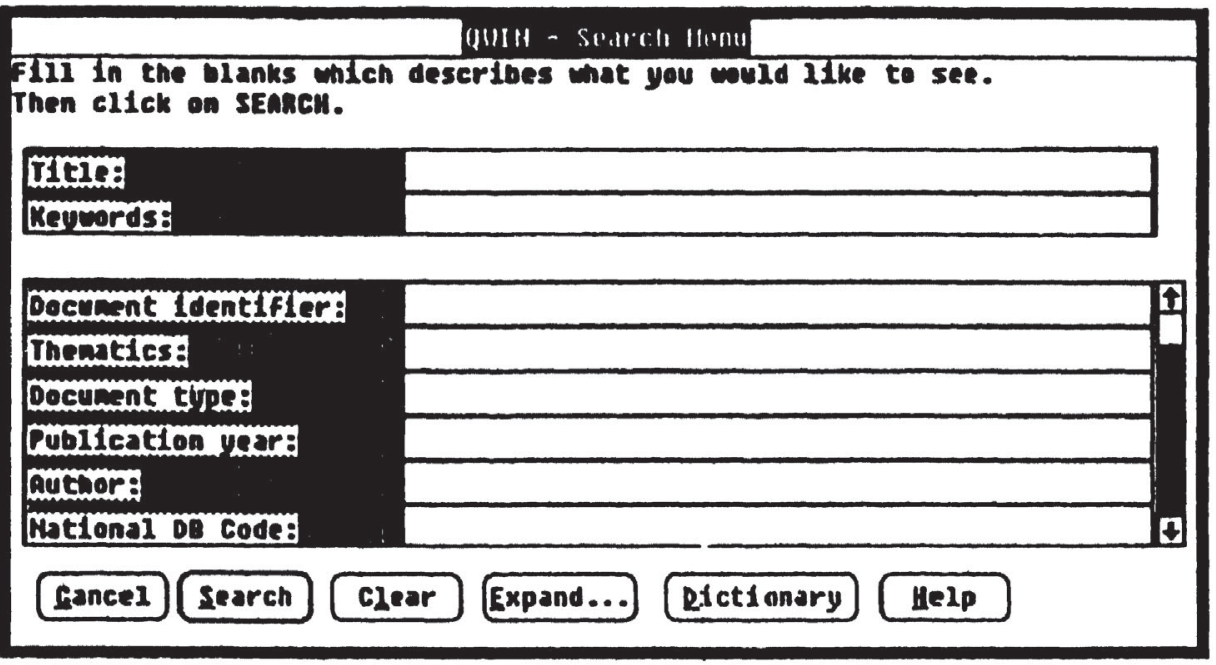

empty area ("free form») in which to enter the search query. A «tool-box» located next to the area (or from a pop-up menu) can give the user access to a selection of searchable fields, boolean operators, proximity operators, a list of neighbouring terms, and other items that are allowed within a search query. In this way, the user may enter a search query using the basic QUEST language (for example). The system will provide the insertion of the correct codes in the search query when the user selects any of the items available from the "tool-box".

The search queries have to be entered observing certain syntactic and semantic rules. The system will attempt to give meaningful feedback when errors are encountered. In its simplest form, this would merely provide the aided recall of certain codes and the ability to cut-and-paste terms and references to the search query, more or less like the current system. The advantage is that the user is free to formulate any combination of field-restricted queries, and to let one and the same query relate to a number of different fields. The disadvantage of this approach is that the user interface gets more difficult to inderstand untuitively.

By offering this possibility in addition to the strictly forms-based approach, one has a system that can "grow" with the user. A beginner will typically start off by using the forms and later advance to using the free-form input, which gives greater flexibility but is more complicated to learn.

The Query-by-Form approach is useful for non-expert users of EsaQuest, because it demands less system knowledge from the user than the basic EsaQuest query language. Some flexibility is sacrificed when using QWIN. The "metaknowledge» about the databases themselves still has to be supplied by the user. Some of this knowledge may be included in QWIN in a pasive manner, for example as part of the help function. Inclusion of active and intelligent help in formulating search queries has not yet been investigated in the context of QWIN.

It becomes increasingly difficult to use QWIN efficiently with increasing numbers of fields, since they either have to be physically located on the screen, or 
Figure 8

QWIN: free- and fixed-text operators
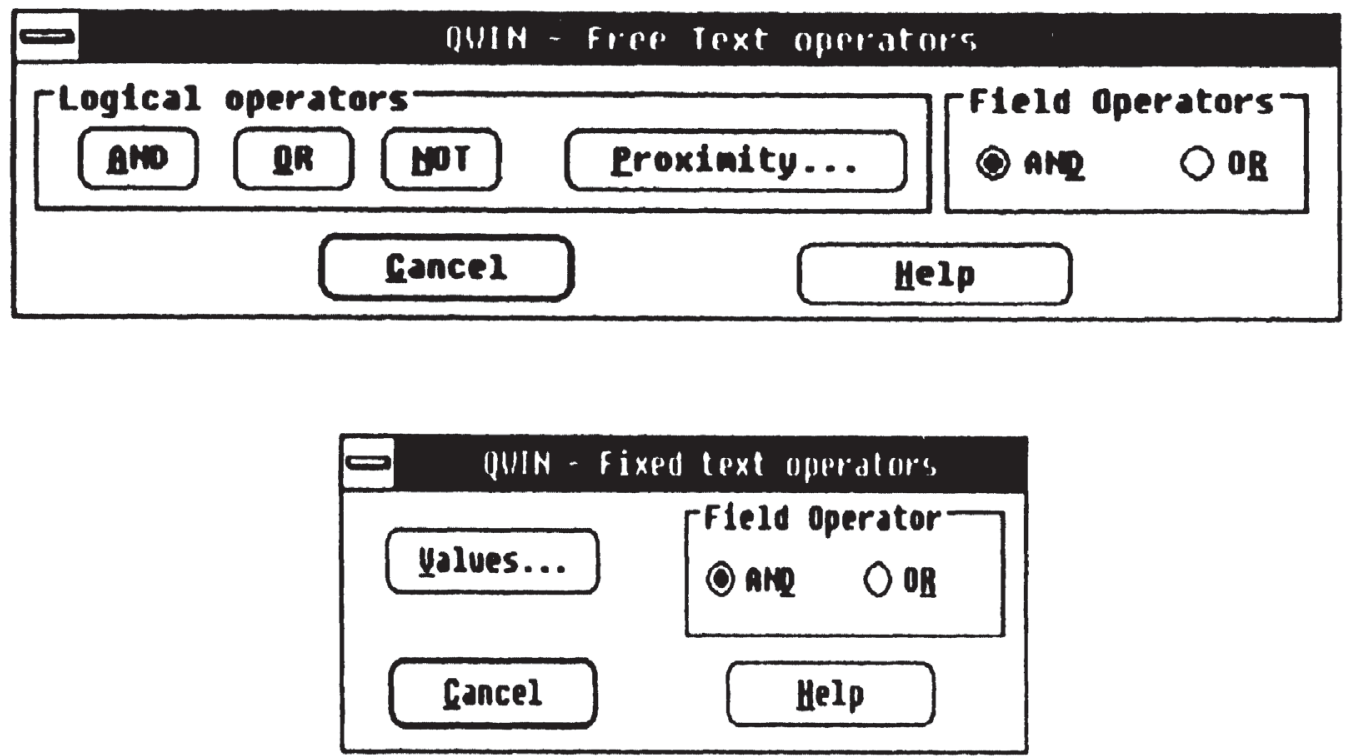

scrolled into view. Later designs may incorporate a means of encapsulating several fields into one entity, which will provide a better overview of the fields available. The user may also be able to select which fields are to be shown, and determine how those fields are ordered.

The proposed "free-field" approach will provide increased flexibility in the formulation of search queries. The interface will give less «intuitive» information, and will thus be more difficult to use, and the syntax rules for the search queries will be more complicated. This method is therefore better suited for the experienced users. The forms-based method and the "free-from» method could be combined, giving a system that can grow with user needs and capabilities.

\section{Conclusion}

In the preceding sections, a number of user-interface projects at ESRIN have been described, indicating their general structure, design principles and methods and, to varying degrees, details of their implementation. Although the projects differ in a variety of aspects, we have attempted to demonstrate some significant ways in which they fit together. In particular, we have noted that an underlying theme is that of user goal and task analysis at a variety of levels as the basis for design of the interface and, more generally, information-system functionality.

The levels of analysis demonstrated by these projects range from overall goals and domain views of specific classes of end users, through specification of goals and tasks of heterogeneous users within information-retrieval systems in general, to goals and characteristics of users within specific information-retrieval systems or databases. An important result of the ESA/ESRIN Workshop was the recognition 
of this type of approach to information-system and information-system-interface design, and specially of the necessity and utility of this approach in the variety of contexts represented by the projects.

This type of analysis led to another important Workshop conclusion, namely that information systems for end users must support a variety of goals and tasks, but through some common interface or seamless access mechanism to a variety of relevant information sources and system functionalities.

The projects described have demonstrated how such systems might be constructed; that is, the types of abstract analysis required, and details of how the results of such analysis lead to the implementation level. However, there are still important problems to be addressed within this approach.

One such problem is the issue of how to actually integrate the various kinds of information sources and functionalities within a single - from the user's viewpoint - environment. One possible approach, supported by the Workshop discussion, is to establish a hierarchy of user goals, starting with goals at the level of, say, the scientific or professional aims of the user, and moving progressively down through goals associated with the resolution of problems, goals associated with information support for problem resolution, goals associated with interaction within information systems, to goals associated with specific information-system functionality. At each of these levels, analysis and representation of tasks and domain views can take place. At any one level, the type of analysis and representation will depend upon that specific context, as for instance when using domain representation in the ESIS project, and Cognitive Task Analysis in the Intelligent Interface for Bibliographic IR project.

Integration is provided by explicitly relating the goal analysis at any one level to one or more tasks or domain characteristics in the level above. This type of analysis could allow the embedding of different information-system functionalities and databases within a single, problem-oriented interface. This kind of multi-level design appears to offer both the kind of modularity required for sufficiently detailed analysis, and a means for maintaining consistency throughout the system. It also suggests that one way of approaching the problem of heterogeneous databases is through an interface based on an abstract problem/domain specification, rather than through database integration.

Beyond these design-oriented themes, the Workshop also had the result of clarifying the general parameters for what we might term "next-generation scientific information systems». It is generally agreed that these will be systems for direct use by end users, which will be capable of supporting heterogeneous user populations in direct response to the problems and tasks on which they are engaged, using the entire variety of information sources required for problem resolution, and integrating all of the information-manipulation functionalities required for the accomplishment of user goals. This is a tall order, and one that can be considered as an ideal goal at which to aim. The Workshop also demonstrated, however, that significant progress is being made at ESRIN towards this goal. 


\section{Acknowledgements}

The authors would like to thank the Head of ESRIN, Mr. F. Roscian, for having made the Workshop on "User Interfaces for Information Systems» possible, and the heads of the projects reported on here for their cooperation.

\section{References}

1. ALBRECHT, M. A.; RUSSO, G.; RICHMOND, A.; HAPGOOD, M. A., Towards a European Space Information System, vol. 1: General Review of User Requirements, ESA Internal Report, 1988.

2. ALBRECHT, M. A.; BODINI, A., Towards a European Space Information System. vol. 2: Implementation Plan, ESA Internal Report, 1988.

3. CATARCI, T.; SANTUCCI, G., Query by Diagram: A Graphical Query System, Proc. 7th Int. Conf. on ER Approach. North-Holland Elsevier, 1988.

4. PAZIENZA, M. T.; FUSCO, L., ESARS: An expert system for accessing remotely sensed data, Proc. Computational Intelligence '88. Univ. degli Studi di Milano (Ed. G. Valle), North-Holland, 1988.

5. PAZIENZA, M. T.; BASILI, R., A natural language interface to expert systems based on logic predicates, modelling the innovation, Proc. TC-7 IFIP Int. Conf.. North Holland Elsevier, 1990.

6. EGAN, D. E., et al., Formative design-evaluation of Super-Book, ACM Trans. Info. Sys., vol. 7, 30-57, 1989.

7. ROTH, E. M.; WOODS, D. D., Cognitive Task Analysis. An approach to knowledge acquisition for intelligent system design, in Topics in Expert System Design: Methodologies and Tools (Eds. G. Guida and C. Tasso), North-Holland, 233-264, 1989.

8. DANIELS, P. J.; BROOKS, H. M.; BELKIN, N. J., Using problem structures for driving human-computer dialogues, in RIAO '85, Actes Conf. Recherche d'Informations Assistee par Ordinateur, Grenoble, March 1985, 131-149, 1985.

9. BELKIN, N. J., et al., Distributed expert-based information systems. An interdisciplinary approach, Information Processing and Management, 23, 395-409, 1987.

10. BELKIN, N. J.; SEEGER, T.; WERSIG, G., Distributed expert problem treatment as a model for information system analysis and design, J. Information Sci., 5, 153-167, 1983.

11. BELKIN, N. J.; BROOKS, H. M.; DANIELS, P. J., Knowledge elicitation using discourse analysis, Int. J. Man-Machine Studies, 27, 127-144, 1987.

12. BELKIN, N. J.; MARCHETTI, P. G., Determining the functionality and features of an intelligent interface to an information retrieval system, Proc. 13th Int. Conf. on Research and Development in Information Retrieval, 1990, Brussels.

13. POLLIT, A. S., A Rule-Based System as an Intermediary for Searching Cancer-Therapy Literature on MEDLINE, in Intelligent Information Systems (Ed. R. Davies), Ellis Howood Ltd., 1986

14. THOMPSON, D. A., Interface Design for an Interactive Information Retrieval System: A Literature Survey and Research System Description, J. Amer. Assoc. Information Sci., 22, 361-372, 1971.

15. GREENBLATT, D.; WAXMAN, J., Datahases: Improving Usability and Responsiveness (Ed. B. Shneiderman), 1978, Academic Press.

16. MCALPINE, G.; INGWERSER, P., Integrated Information Retrieval in a Knowledge Worker Support System, Proc. 12th Int. Conf. on Research and Development in Information Retrieval, 1989, Cambridge, USA. 